\title{
Determinación simplificada del contenido de lignina en paja de trigo por método volumétrico y su caracterización química
}

\author{
Lydia Toscano Palomar ${ }^{1}$, Alan Gabriel Ayala Bautista ${ }^{2}$, Gisela Montero \\ Alpirez ${ }^{3}$, Lourdes Cervantes Díaz ${ }^{4}$, Ricardo Torres Ramos ${ }^{5}$, Erick Romero \\ Uscanga ${ }^{6}$, María Guadalupe Amado Moreno ${ }^{7}$ y Ángel García Velázquez ${ }^{8}$
}

\section{Resumen}

El uso de biomasa lignocelulósica como fuente de energía renovable se ha convertido progresivamente en un tema fundamental. Grandes esfuerzos se están realizando enfocados en la identificación y caracterización de biomasa adecuada que pueda ser utilizada para el reemplazo de combustibles fósiles convencionales. El principal objetivo del presente estudio es determinar la correlación que existe entre los parámetros del No. Kappa y el contenido de lignina en la paja de trigo y hacer un análisis químico de la paja de trigo que queda como residuo agrícola del cultivo de Trigo en el Valle de Mexicali, México; todo esto para su futuro uso como biocombustible de siguiente generación. Se realizaron los análisis de Humedad, cenizas, Proteína cruda, carbono orgánico, extraíbles en hexano, extraíbles en acetona, extraíbles en agua, No. Kappa, lignina, hemicelulosa y celulosa. Las muestras de paja de trigo fueron posteriormente caracterizadas por Espectroscopia de infrarrojo por transformadas de Fourier para la asociación del contenido de lignina con los resultados reportados. Un factor de correlación de 0.239 entre el No. Kappa y el contenido de lignina se determinó en base a manejo de datos para diferentes tipos de muestras de paja de trigo con la finalidad de poder determinar el contenido de lignina por un método de análisis más sencillo y rápido para estudios posteriores de delignificación de la paja del trigo.

Palabras clave: Lignina, No. Kappa, Paja de trigo, Biomasa.

1 Profesora Investigadora, Departamento de Ingeniería Química, Instituto Tecnológico de Mexicali.

2 Estudiante de Posgrado, Departamento de Ingeniería Química, Instituto Tecnológico de Mexicali.

3 Profesora Investigadora, Instituto de Ingeniería, Universidad Autónoma de Baja California, México.

4 Profesora Investigadora, Instituto de Ciencias Agrícolas, Universidad Autónoma de Baja California, México.

5 Estudiante de Posgrado, Instituto de Ingeniería, Universidad Autónoma de Baja California, México.

6 Estudiante de Posgrado, Instituto de Ingeniería, Universidad Autónoma de Baja California, México.

7 Profesora Investigadora, Departamento de Ciencias Básicas, Instituto Tecnológico de Mexicali, México.

8 Profesor Investigador, Departamento de Ciencias Básicas, Instituto Tecnológico de Mexicali, México. 


\begin{abstract}
The use of lignocellulosic biomass as a renewable energy source has gradually become a major issue. Great efforts are being made focused on the identification and characterization of suitable biomass which can be used to replace conventional fossil fuels. Selection of this biomass requires reliable and rapid methods for compositional characterization of plant material. Many standard analytical methods are laborious and slow. The use of simpler and more rapid methods have proved to be invaluable in biomass analysis. The main objective of this study is to determine the correlation between the parameters of Kappa number and the Klason lignin content in different samples of wheat straw with the purpose of determining the lignin content by a simpler and quicker method of analysis. The biomass was examined physiochemically to understand its compositional characteristics through analytical approaches such as gravimetric and volumetric methods. The results indicate 0.239 as a correlation factor between Kappa number y Klason lignin content for wheat straw. Further, Fourier transform infrared spectroscopy was used to indicate characteristic absorption bands of the constituents in the different used samples.
\end{abstract}

Keywords: Lignin, No. Kappa, Wheat straw, Biomass 


\section{Introducción}

Las Fuentes de Energías Renovables (FER) se han convertido en tema de prioridad en muchos países debido a la necesidad de reducir las emisiones de gases de efecto invernadero y por consecuencia el problema del calentamiento global. Por lo tanto, se consideran una alternativa positiva al uso de combustibles fósiles y sus derivados (Ahiduzzaman, Aminul Islam, Yaakob, Ghani, \& Anuar, 2014). La biomasa abarca toda la materia orgánica de origen vegetal o animal y representa ser una prometedora FER. La biomasa a partir de las plantas vegetales es un producto de la fotosíntesis y puede ser considerado un carbón neutral en el ciclo de vida de esta (Kim \& Dale, 2005). Entre los residuos agrícolas particularmente atractivos por su bajo costo y alta disponibilidad se encuentran la paja de arroz, mazorca, rastrojo de maíz, bagazo, paja de trigo, etc. Entre muchos de los residuos agrícolas, la paja de trigo es el segundo material lignocelulósico más favorable, después de la paja de arroz en términos de su disponibilidad y abundancia (Kim \& Dale, 2004). Los tres principales componentes de la biomasa a partir de plantas son la celulosa, hemicelulosa y lignina. La celulosa es el principal componente de la pared celular en las plantas y es un polímero regular de alto peso molecular, formado por unidades de glucosa. Las hemicelulosas son un grupo de polisacáridos compuestos de pentosas y hexosas. El tercer más importante componente es la lignina, conformado de tres diferentes unidades monoméricas de fenilpropanoides (alcoholes de sinapilo, coniferilo y $p$-coumarilo) los cuales se polimerizan por acoplamiento de radicales después de una oxidación enzimática, dando como resultado una macromolécula sin una estructura definida (Ralph, Lundquist, Brunow, Lu, Kim \& Schatz, 2004). La biomasa a partir de residuos de plantas es generalmente no comestible. La biomasa lignocelulósica se encuentra químicamente compuesta de 35-55\% en celulosa, 20-40 \% hemicelulosa y de 10-25\% en lignina. El tipo de la biomasa y sus propiedades intrínsecas determinan tanto la selección del proceso de conversión como la subsecuente complejidad del proceso y también explica la cantidad de energía almacenada en ella. La composición de los azúcares (pentosas y hexosas) define el rendimiento teórico de biocombustible y puede por lo tanto tener un impacto considerable en el análisis de costo y beneficio (McKendry, 2002). Un aspecto primario en el uso de la biomasa como biocombustible es el conocimiento de su composición básica y propiedades. La caracterización cualitativa y cuantitativa de sus componentes es esencial para las perspectivas de su aplicación. Por lo tanto, una caracterización general de la biomasa es indispensable para la expansión de los sectores en bioenergía y bioproductos en todo el mundo.

De los diferentes métodos para la determinación de lignina en plantas, el uso de acido sulfúrico al $72 \%$ para la hidrólisis de todos los carbohidratos conocido como lignina Klason ha sido ampliamente utilizado. El método es laborioso, tardado 
y puede arrojar resultados erróneos debido a hidrólisis incompleta del contenido celulósico (Soo-Jeong, Nam-Seok \& Yuan-Zong, 2007). La estimación del número Kappa es un método utilizado para determinar el grado de delignificación en la pulpa de madera el cual es una medida del volumen de permanganato de potasio consumido por un gramo de muestra bajo condiciones estándar de análisis (TAPPI T236, 1999). El objetivo del presente estudio fue caracterizar la paja de trigo y desarrollar el método más sencillo y rápido para la estimación del contenido de lignina en la paja de trigo, relacionando su valor con el del número Kappa.

\section{Materiales y Métodos}

\section{Biomasa}

La paja de trigo (Triticum aestivum) utilizada fue obtenida de agricultores locales del cultivo de invierno. Fue secada al ambiente por un período de 2 meses y lavada para remover tierra y suciedad, secada al ambiente por 2 semanas y molida en un molino de cuchillas. La biomasa triturada se separó por tamaño de partícula. Se colectaron las partículas que se retuvieron en las mallas \#16 $(1.18 \mathrm{~mm})$, \#18 (1.0 $\mathrm{mm})$, \# $25(0.7 \mathrm{~mm})$, \# $35(0.5 \mathrm{~mm})$ y \# $40(0.42 \mathrm{~mm})$. Las partículas que pasaron la malla \# 40 se colectaron para futuras experimentaciones. Las muestras pulverizadas se almacenaron en bolsas de plástico y utilizadas como se fue necesitando. La edad del material después de colectarse, secarse y molerse fue alrededor de 2-4 meses hasta el momento de su análisis. Las muestras utilizadas para la determinación de la correlación entre el No. Kappa y el contenido de lignina fueron paja cruda, pretratada álcali (hidróxido de sodio 1\%), pretratada ácido-base (ácido sulfúrico $1 \%$ e hidróxido de sodio $1 \%$ ) y paja blanqueada (clorito sódico).

\section{Análisis Próximo}

El tratamiento de la muestra, los contenidos de humedad, cenizas, proteína cruda, lípidos, extraíbles a la acetona y extraíbles al agua caliente en muestras de paja se determinaron usando procedimientos estándares descritos en TAPPI T257, TAPPI T412 om-02, TAPPI T211, NRL/TP-510-42625, AOAC 920.39, TAPPI T204 cm-97 y TAPPI T207 cm-99 respectivamente.

\section{Análisis Químico}

La paja de trigo seca y molida se trató de acuerdo al procedimiento estándar descrito en TAPPI T264 cm-07 para determinar los contenidos de lignina insoluble en ácido, 
alfa,-beta- y gama celulosa, No. Kappa, se siguieron las normas TAPPI T222 om-02, TAPPI T203 cm-99 y TAPPI T236 om-99, respectivamente. El contenido de carbono orgánico total fue medido por el método de Nelson y Sommers, 1982. El contenido de holocelulosa se determinó por tratamiento de paja libre de extractivos con clorito de sodio $(\mathrm{NaClO} 2)$ y ácido acético $(\mathrm{CH} 3 \mathrm{COOH})$ (Rabemanolontsoa \& Saka, 2011).

\section{Pretratamiento de la muestra}

Para fines de obtener la relación del contenido de lignina con el valor de No. Kappa la paja de trigo seca, molida (malla \#12) fue sometida a cuatro diferentes pretratamientos: Básico (TB) con solución de $\mathrm{NaOH}$ al 1\% w/v de acuerdo a la norma estándar TAPPI T212, Ácido-Básico (AB) con solución H2SO4 al 0.75\% $\mathrm{v} / \mathrm{v}$, por 2 horas, filtrar y lavar con agua destilada para remover el ácido, después se agrega solución de $\mathrm{NaOH} 1.5 \%$ w/v durante la noche y filtrando. La paja tratada se lava hasta $\mathrm{pH}$ neutro y se deja secar. El tercer y cuarto tratamientos consistieron en tratamientos ligeramente blanqueado (LB) y semiblanqueado (SB). Los sólidos fueron ligeramente blanqueados a $70 \pm 2{ }^{\circ} \mathrm{C}$ en un baño de agua con una relación inicial de líquido-sólido de 30 y una adición de clorito de sodio $0.6 \mathrm{~g} / \mathrm{g}$ de biomasa seca y de ácido acético $0.6 \mathrm{ml} / \mathrm{g}$ de biomasa seca. Se procedió de igual manera para el semiblanqueado, con dos aplicaciones de $\mathrm{NaClO} 2$ y CH3COOH. La mezcla se mantuvo en campana de extracción de vapores, con agitación intermitente por períodos de dos horas entre adiciones de clorito de sodio y ácido acético. Después del tratamiento, la mezcla se filtró en un filtro de vidrio para separar el líquido de los sólidos. Los sólidos se lavaron repetidamente con agua desionizada a temperatura ambiente hasta un $\mathrm{pH}$ cerca de la neutralidad. Los sólidos se llevaron a secar y se colectaron para experimentaciones posteriores. La Figura 1 muestra el aspecto de las muestras de paja de trigo tratadas que se utilizaron en este estudio.

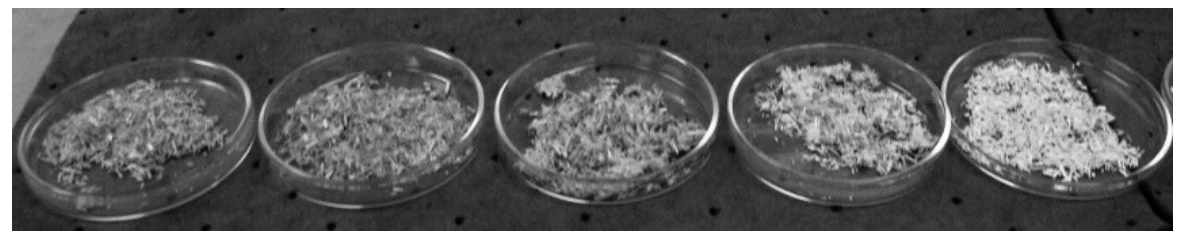

Fig. 1 Muestras de biomasa analizadas en este estudio. De izquierda a derecha, Paja de trigo sin tratar, con Tratamiento Básico (TB), Tratamiento Ácido-Básico (AB), Tratamiento Ligeramente Blanqueado (LB) y Tratamiento Semiblanqueado (SB). 


\section{Lignina insoluble en ácido (Lignina Klason)}

La paja molida (malla \# 12) libre de extraíbles se sometió a hidrólisis ácida con solución de H2SO4 (72\% w/w). Después de dispersar el material en el ácido a $20 \pm 1{ }^{\circ} \mathrm{C}$, se mantuvo en baño a esta temperatura por 2 horas con agitación frecuente. Se continuó la hidrólisis del material diluyendo el H2SO4 $(3 \% \mathrm{w} / \mathrm{w})$ por adición de agua desionizada y ebullición a reflujo por 4 horas. Las muestras se filtraron y el residuo sólido se secó y pesó para la determinación de la lignina Klason.

\section{Número Kappa}

Este método se aplica para la determinación del grado de delignificación de pulpas no blanqueadas y semiblanqueadas obtenidas hasta un rendimiento por debajo del 70\%. La prueba se realizó en muestras secas y molidas (malla \# 25) con permanganato de potasio $\left(\mathrm{KMnO}_{4} 0.1 \mathrm{~N}\right)$ en ambiente ácido con $\mathrm{H}_{2} \mathrm{SO}_{4}$ de acuerdo con método clásico TAPPI T236 om-99. Las muestras se analizaron por triplicado. La correlación entre el No. Kappa y el contenido de lignina se determinó por el análisis de los datos experimentales y por correlación lineal.

\section{Espectroscopia de IR (FTIR)}

Espectros de infrarrojo por transformadas de Fourier (FTIR) de las diferentes muestras de paja utilizadas durante este estudio se registraron en un Espectrofotómetro FTIR Rayleigh WQF-510. Las muestras se prepararon en pastillas de bromuro de potasio $(\mathrm{KBr})$ por presión de una mezcla de $2 \mathrm{mg}$ de muestra con $400 \mathrm{mg}$ de $\mathrm{KBr}$ en una prensa. Para eliminar interferencia por presencia de humedad, las muestras y el $\mathrm{KBr}$ fueron deshidratados separadamente $105^{\circ} \mathrm{C}$ antes de formar el comprimido. Los espectros se realizaron en un rango de frecuencias de 600 a $4000 \mathrm{~cm}^{-1}$ con resolución de $4 \mathrm{~cm}^{-1}$ y 30 escaneos. La línea base fue corregida y normalizada en transmitancia.

\section{Resultados}

En este estudio se evaluó la paja de trigo de la variedad RAFI C-97, representa los tipos de cultivo invierno y primavera. Su morfología incluye paja de tipo sólida y hueca. El material lavado y seco fue molido $(>0.42 \mathrm{mM})$. La paja se analizó por triplicado para humedad, extractivos al agua caliente, extractivos a la acetona, lignina insoluble, número Kappa, alfa-,beta- y gama celulosa, proteína cruda, carbono orgánico total, lípidos y cenizas. En la Tabla1 se tabulan los valores. Valores similares en contenidos de humedad, cenizas, lignina y proteína cruda fueron reportados por Ahmed, Zia \& Iqbal, 2010 y Khan \& Mubeen, 2012. 
Tabla 1. Composición de la paja de trigo

\begin{tabular}{|c|c|c|c|c|}
\hline & & & \multicolumn{2}{|c|}{ Otras Referencias } \\
\hline No. & Componente (\% Peso seco) & Propios & $\begin{array}{c}\text { Ahmed et al, } \\
\mathbf{2 0 1 0}\end{array}$ & $\begin{array}{c}\text { Khan \& } \\
\text { Mubeen,2012 }\end{array}$ \\
\hline $\mathbf{1}$ & Humedad & $5.35 \pm 0.2$ & $5.07 \pm 0.24$ & 7.3 \\
\hline $\mathbf{2}$ & Cenizas & $8.30 \pm 0.3$ & $7.30 \pm 0.42$ & 6.0 \\
\hline $\mathbf{3}$ & Extractivos a la acetona & $5.20 \pm 0.2$ & - & 9.5 \\
\hline $\mathbf{4}$ & Extractivos al agua caliente & $15.45 \pm 0.2$ & - & - \\
\hline $\mathbf{5}$ & Extractivos al hexano (Lípidos) & $4.51 \pm 0.3$ & $1.85 \pm 0.02$ & - \\
\hline $\mathbf{6}$ & Lignina insoluble & $14.85 \pm 1.0$ & $6.40 \pm 0.19$ & 15.0 \\
\hline $\mathbf{7}$ & $\alpha$-Celulosa & $34.76 \pm 0.4$ & $39.40 \pm 0.72$ & 38.0 \\
\hline $\mathbf{8}$ & $\beta$-Celulosa & $1.67 \pm 0.02$ & - & - \\
\hline $\mathbf{9}$ & $\gamma$-Celulosa & $8.55 \pm 0.05$ & - & 29.0 \\
\hline $\mathbf{1 0}$ & Proteína cruda (NT $\times 6.25)$ & $1.30 \pm 0.01$ & $3.50 \pm 0.13$ & 4.0 \\
\hline $\mathbf{1 1}$ & Carbono orgánico Total (COT) & $35.5 \pm 0.4$ & - & - \\
\hline
\end{tabular}

Los valores de lignina, número Kappa y Kappa lignina para cinco muestras diferentes de paja de trigo se muestran en la Tabla 2. Los valores más elevados se encontraron para la muestra con el tratamiento álcali-ácido y los valores menores en la muestra químicamente modificada por el semiblanqueado.

Tabla 2. No. Kappa, lignina Klason y lignina Kappa de muestras de paja de trigo diferentes

\begin{tabular}{|c|c|c|c|}
\hline Muestras & No. Kappa & $\begin{array}{c}\text { Lignina Klason } \\
\mathbf{( \% )}\end{array}$ & $\begin{array}{c}\text { Lignina Kappa } \\
\mathbf{( \% )}\end{array}$ \\
\hline Sin tratamiento & 74.95 & 17.82 & 18.23 \\
\hline Tratada con álcali & 49.43 & 13.15 & 12.24 \\
\hline Tratada con álcali-ácido & 46.99 & 11.36 & 11.67 \\
\hline Blanqueado ligero & 24.79 & 6.98 & 6.46 \\
\hline Semiblanqueada & 17.77 & 4.10 & 4.81 \\
\hline
\end{tabular}

Los resultados de los contenidos de lignina Klason y número Kappa de las muestras de paja de trigo sin tratar y químicamente tratadas se muestran en la Figura 2. De esta gráfica se observa que el número Kappa de la paja de trigo presenta una relación lineal con su correspondiente lignina Klason determinándose que la correlación que existe entre ambas es de 0.239 la cual es el resultado de la pendiente de la recta descrita. 


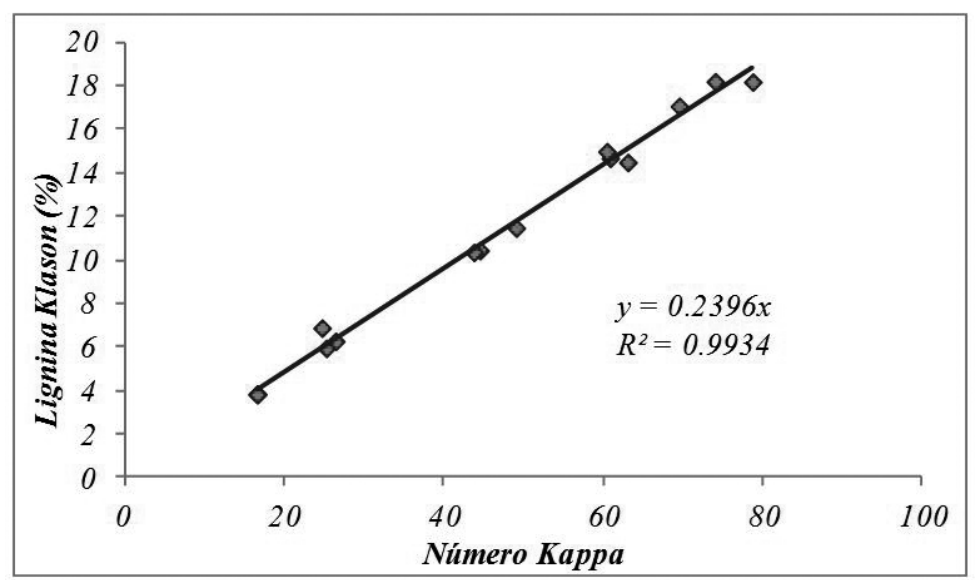

Fig. 2 Relación del contenido de Lignina Klason y Número Kappa en muestras de paja de trigo tratadas y sin tratar. Valor de correlación 0.239

Espectroscopia de FTIR se utilizó para identificar grupos funcionales presentes en las muestras. Los mismos picos de absorbancia se identificaron en las muestras de paja tratadas y sin tratar solo con diferencias en las intensidades, Figura 3. Se observan picos o bandas de absorción a los 3450, 1740, 1600-1650 y 1110-1050 $\mathrm{cm}^{-1}$, lo que muestra presencia de compuestos aromáticos, fenólicos, alifáticos $\mathrm{y}$ estructuras polisacáridas. Una banda de absorción ancha a los $3405 \mathrm{~cm}^{-1}$ es atribuida a grupos oxidrilos que normalmente ocurren como resultado de la asociación entre los polímeros. La intensidad de esta banda es influenciada por la concentración de la muestra (Sun, Sun, Fowler \& Baird, 2005).

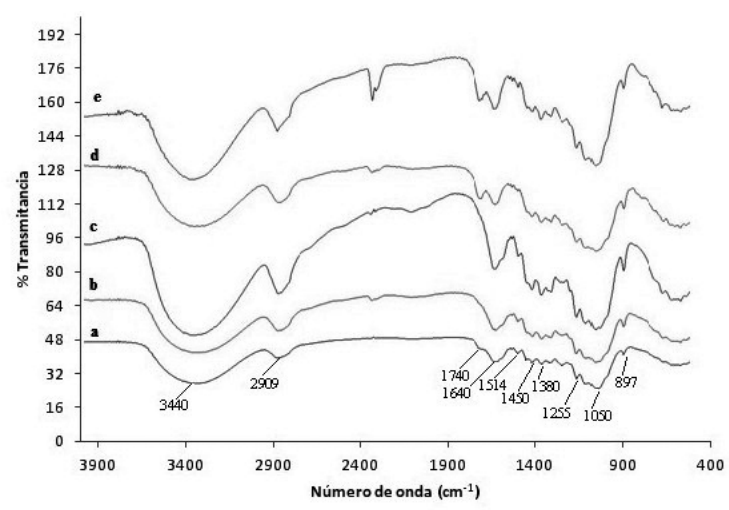

Fig. 3 Espectros de FTIR de muestras de paja de trigo (a) sin tratar, (b) tratamiento básico TB, (c) tratamiento ácido-básico AB, (d) ligeramente blanqueada y (e) Semiblanqueada. 
Se observó una banda a $2910 \mathrm{~cm}^{-1}$ indicando vibraciones de estiramiento por enlaces $\mathrm{C}-\mathrm{H}$ debido a grupos $\mathrm{CH} 2$ y $\mathrm{CH} 3$; confirmándose estos grupos por vibraciones de flexión en el $1380 \mathrm{~cm}^{-1}$ presentes en las estructuras de la celulosa y hemicelulosa (Peng et al, 2009). Bandas de absorción entre 1125 y $1000 \mathrm{~cm}^{-1}$ son típicas de presencia de xilanos, debidas al estiramiento de $\mathrm{C}-\mathrm{O}$ y C-C y a la contribución del enlace glucosídico. Bandas muy delgadas en $\operatorname{los} 890 \mathrm{~cm}^{-1}$ se atribuye a los enlaces $(1 \rightarrow 4) \beta$-glucosídico entre unidades de xilosa en la hemicelulosa (Sun, Sun, Fowler \& Baird). La presencia de la lignina se observa por una banda característica en $1525-1510 \mathrm{~cm}^{-1}$.

\section{Conclusiones}

Este estudio demostró la correlación que existe entre el contenido de lignina y la determinación del No. Kappa en la paja de trigo. Otros estudios similares realizados en pulpas y maderas han reportado factores de correlación entre $0.13 \mathrm{y}$ 0.15 . En el caso de pajas y forrajes se han hecho estudios de correlación entre el contenido de lignina y otros componentes como el contenido de proteína utilizando otro método de análisis de lignina como la degradación de fibras en detergente neutro (NDF) (Gomez et al, 2011). Jung, Varel, Weimer \& Ralph, 1999 estimaron el valor calorífico correlacionando el contenido de lignina Klason y Lignina al detergente ácido (ADL). De acuerdo con la relación No. Kappa-Lignina estimada (0.239) el No. Kappa de las diferentes muestras se multiplicó por este valor dando indirectamente el contenido de lignina en las muestras, las cuales se muestran en la Tabla 2 bajo la columna nombrada como Lignina Kappa para distinguirla de la Lignina Klason. 


\section{Referencias}

Ahiduzzaman, M., Aminul Islam, A. K., Yaakob, Z., Ghani, J.A. \& Anuar, N. (2014). Agricultural residues from crop harvesting and processing: A renewable source of Bio-Energy, in Biomass and Bioenergy Applicatons, K.R. Hakeem and M. Jawaid, Rashid, U., Editors. Springer. p. 323-337. DOI 10.1007/9783-319-07578-5_16

Ahmed, I., Zia, M.A. \& Iqbal, H. M. (2010). Bioprocessing of Proximally Analyzed Wheat Straw for Enhanced Cellulase Production through Process Optimization with Trichoderma viride under SSF. International Scholarly and Scientific Research \& Innovation, 4(1): p. 1028-1034. http://waset.org/ publications $/ 14480$

Gomez, D.I., E. Detmann, Valadares Filho, S., Fukushima, R.S., Souza, M.A., Valente, T., Paulino, M.F. \& Queiroz, A.C. (2011). Evaluation of lignin contents in tropical forages using different analytical methods and their correlations with degradation of insoluble fiber. Animal Feed Science and Technology, 168(3-4): p. 206-222. DOI:10.1016/j.anifeedsci.2011.05.001

Hames, B., Scarlata, C., \& Sluiter, A. (2008). Determination of protein content in biomass. National Renewable Energy Laboratory: Golden, CO. http://www. nrel.gov/biomass/pdfs/42625.pdf

Jung, H.G., Varel, V.H., Weimer, P.J. \& Ralph, J. (1999). Accurancy of Klason Lignin and Acid Detergent Lignin Methods as assessed by Bomb Calorimetry. J. Agric. Food Chem., 47(5): p. 2005-2008. DOI: 10.1021/jf981250q

Khan, T.S. \& Mubeen, U. (2012). Wheat Straw: A Pragmatic Overview. Current Research Journal of Biological Sciences, 4(6): p. 673-675.

http://maxwellsci.com/print/crjbs/v4-673-675.pdf

Kim, S. \& Dale, B.E. (2004). Global potential bioethanol production from wasted crops and crop residues. Biomass and Bioenergy, 26(4): p. 361-375. http:// www.sciencedirect.com/science/article/pii/S0961953403001375

Kim, S. \& Dale, B.E. (2005). Life cycle assessment of various cropping systems utilized for producing biofuels: Bioethanol and biodiesel. Biomass and Bioenergy, 29(6): p. 426-439. DOI:10.1016/j.biombioe.2005.06.004

McKendry, P. (2002). Energy production from biomass (part 1): overview of biomass. Bioresource Technology., 83: p. 37-46. DOI:10.1016/S09608524(01)00118-3 
Nelson, D.W. \& Sommers, L. (1982). Total carbon, organic carbon and organic matter in Methods of soil analysis Part 2. Chemical and microbiological properties, (methodsofsoilan2), p. 539-579.DOI:10.2134/agronmonogr9.2.2ed. c29

Peng, F., J.L. Ren, Xu, F., Bian, J., Peng, P., \& Sun, R. C. (2009). Comparative study of hemicellulose obtained by graded ethanol precipitation from sugarcane bagasse. Journal of Agricultural and Food Chemistry, 57(14): p. 6305-6317.

DOI: $10.1021 / \mathrm{j} 900986 \mathrm{~b}$

Rabemanolontsoa, H. \& Saka, S. (2012). Holocellulose determination in biomass. In Zero-Carbon Energy Kyoto 2011, p. 135-140. Springer Japan.

DOI: 10.1007/978-4-431-54067-0_14

Ralph, J., Lundquist, K., Brunow, G., Lu, F., Kim, H., Schatz, P. F. (2004). Lignins: natural polymers from oxidative coupling of 4-hydroxyphenyl-propanoids. Phytochemistry Reviews, 3(1-2): p. 29-60.

DOI: 10.1023/B:PHYT.0000047809.65444.a4

Shin, S. J., Cho, N. S., \& Lai, Y. Z. (2007). Residual extractives in aspen kraft pulps and their impact on Kappa number and Klason lignin determination. Journal of Wood Science, 53(6): p. 494-497. DOI: 10.1007/s10086-007-0894-8

Sun, X.F., Sun, R., Fowler, P., \& Baird, M. S. (2005). Extraction and characterization of original lignin and hemicelluloses from wheat straw. Journal of Agricultural and Food Chemistry, 53(4): p. 860-870. DOI: 10.1021/jf040456q

TAPPI, T. (2004). $204 \mathrm{~cm}-97$, Solvent extractives of wood and pulp. Technical Association of the Pulp and Paper Industry, 2005.

http://www.tappi.org/content/sarg/t204.pdf

TAPPI, T. (2002). $211 \mathrm{om}-02$. Ash in Wood and Pulp. Technical Association of the Pulp and Paper Industry, 2003.

http://research.cnr.ncsu.edu/wpsanalytical/documents/T211.PDF

TAPPI, T. (1999). 236 om-99. Kappa number in pulp. Technical Association of the Pulp and Paper Industry, 2000.

http://research.cnr.ncsu.edu/wpsanalytical/documents/T236.PDF

TAPPI, T. (2002). $257 \mathrm{~cm}-02$. Sampling and Preparing Wood for Analysis. Technical Association of the Pulp and Paper Industry, 2003. 
TAPPI, T. (2008). 550 om-08. Moisture in Pulp, Paper and Paperboard. (2008). Technical Association of the Pulp and Paper Industry, 2012.

http://www.tappi.org/content/tag/sarg/t550.pdf

TAPPI, T. (1999). 203 cm-99. Alpha-,beta- and gamma-cellulose in pulp. Technical Association of the Pulp and Paper Industry, 1999.

http://research.cnr.ncsu.edu/wpsanalytical/documents/T203.PDF

TAPPI, T. (1999). $207 \mathrm{~cm}-99$. Water solubility of wood and pulp. Technical Association of the Pulp and Paper Industry, 2002.

http://research.cnr.ncsu.edu/wpsanalytical/documents/T207.PDF

TAPPI, T. (2002). 212 om-02. One percent sodium hydroxide solubility of wood and pulp. Technical Association of the Pulp and Paper Industry, 2003.

http://research.cnr.ncsu.edu/wpsanalytical/documents/T212.PDF

TAPPI, T. (2002). 222 om-02. Acid-insoluble lignin in wood and pulp. Technical Association of the Pulp and Paper Industry, 2006.

http://www.tappi.org/content/SARG/T222.pdf

TAPPI, T. (2007). $264 \mathrm{~cm}-07$. Preparation of wood for chemical analysis. Technical Association of the Pulp and Paper Industry.

Thiex, N. J., Anderson, S., \& Gildemeister, B. (2003). Crude fat, Hexanes extraction, in feed, cereal grain, and forage (Randall/Soxtec/Submersion method): Collaborative study. Journal of AOAC International, 86(5): p. 899908.

http://www.ingentaconnect.com/content/aoac/jaoac/2003/00000086/00000005/ $\operatorname{art00005}$ 